\title{
Tracking Electrons and Atoms in a Photoexcited Metalloporphyrin by X-ray Transient Absorption Spectroscopy
}

\author{
Lin X. Chen, ${ }^{a *}$ Xiaoyi Zhang, ${ }^{a, b}$ Erik C. Wasinger, ${ }^{a}$ Klaus Attenkofer, ${ }^{b}$ Guy Jennings, ${ }^{b}$ \\ Ana Z. Muresan, ${ }^{c}$ and Jonathan S. Lindsey ${ }^{c}$ \\ ${ }^{a}$ Chemistry Division and ${ }^{b} X$-ray Science Division, Argonne National Laboratory, Argonne, Illinois 60439, USA \\ ${ }^{c}$ Department of Chemistry, North Carolina State University, Raleigh, North Carolina 27695, USA.
}

RECEIVED DATE (automatically inserted by publisher); Ichen@anl.gov

\section{Supporting Information}

\section{Description of experimental setup and conditions of X-ray transient absorption (XTA) measurements}

The XTA measurements were carried out at Beamline 11ID-D, the Advanced Photon Source of Argonne National Laboratory. ${ }^{1}$ The newly implemented timing scheme of the XTA experiments is shown in Figure 1. The laser pump, X-ray probe cycle was at $1 \mathrm{kHz}$ repetition rate, limited by that of the laser pulses $(1 \mathrm{~mJ} /$ pulse, 5 ps fwhm at $527 \mathrm{~nm}$ ). The $527 \mathrm{~nm}$ light coincides with the Q-band of NiTMP, inducing the $\mathrm{S}_{0} \rightarrow \mathrm{S}_{1}$ transition. The fraction of the excited-state NiTMP molecules was determined to be $45-50 \%$ by the optical transient absorption change of the same sample using the same laser excitation source and sample geometry. The XTA signals were averaged at each X-ray photon energy defined by an X-ray monochromator (with double Si 111 crystals) for $200-400$ seconds. The X-ray probe pulses (approximately $10^{5}$ photons/pulse at $8 \mathrm{keV}, 160 \mathrm{ps}$ fwhm) were extracted from a train of electron bunches circulating in the storage ring at $272 \mathrm{kHz}$ under a hybrid timing mode where an intense X-ray pulse with $16 \%$ of the total average photon flux was separated in time from other weak X-ray pulses with $84 \%$ of the total photon flux by $\sim 1.59 \mu$ s (see Figure 1). Therefore, only $0.06 \%$ of total X-ray photons could be used as the probe at the $1 \mathrm{kHz}$ repetition rate. An eight-element solid-state germanium detector array (Canberra) was used to collect X-ray fluorescence signals with the Soller slit and a cobalt filter (six absorption lengths) combination to remove elastic scattering signal and minimize the cobalt filter

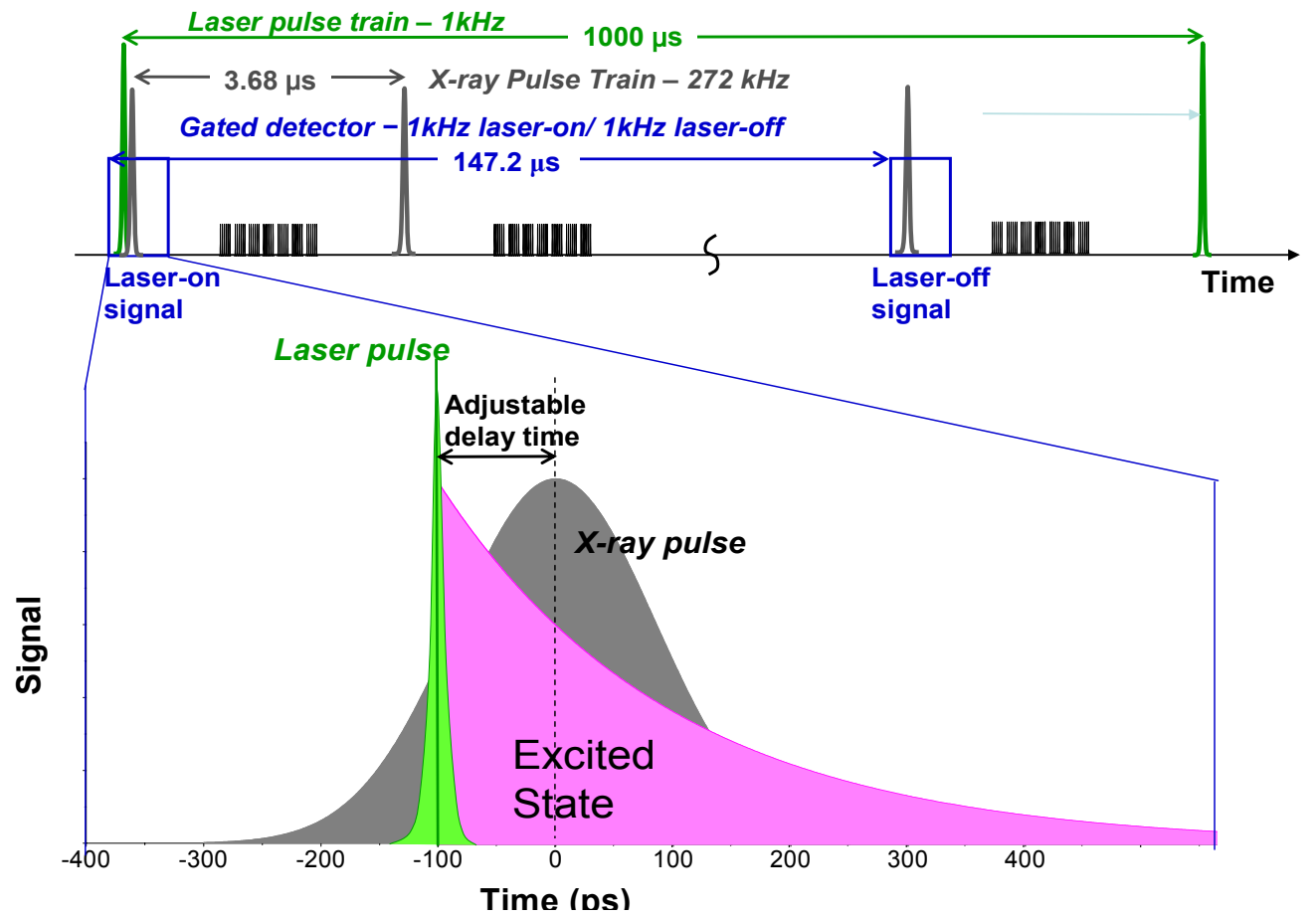

Figure 1. The timing scheme of the XTA experiment. 
fluorescence signals. The detector signals were processed by pre-amplifiers, amplifiers and single channel analyzers (SCAs) with the proper upper and lower thresholds set for the $\mathrm{Ni} \mathrm{K}_{\alpha}$ fluorescence energy. The output signals from the SCAs were split into three portions connecting to three scaler sets, respectively. The first portion was ungated for the ground-state signals, the second portion was electronically gated with the laser pulse for the laser-on signals, and the third portion gated after 40 round trips of the X-ray pulses (147 $\mu$ s later than the laser pulse) for the laser-off signals. Thus the second and the third portions of the signals have the same counting statistics. The laser-off signals were used to construct the ground-state $\mathrm{S}_{0}$ spectrum, and the laser-on signals were used to construct the spectrum of the $T_{1}$ state and the remaining $S_{0}$ state mixture, which was used later to extract the $T_{1}$ state spectrum. The delay time between the laser and the X-ray pulses was adjusted by a programmable delay line (PDL-100A-20NS, Colby Instruments) that adjusts the phase shift of the mode-lock driver for the seed laser relative to that of the RF signal of the storage ring with a precision of $500 \mathrm{fs}$. The delay time as shown in Figure 1 is $100 \mathrm{ps}$, with the X-ray pulse lags the laser pulse. At this delay time, the optimal XTA signals were obtained. XTA spectrum at the delay time $\mathrm{t}=0 \mathrm{ps}$ was also collected and shown in Figure $2 \mathrm{~A}$ in the main text.

2. EXAFS data fitting curves and structural parameter table.

The simulated NiTMP structure based on the crystal structure of NiTPP was used in the FEFF8.0 calculation to obtain the scattering paths with phases and amplitudes of the ground state. The fitting to the experimental data is shown in Figure 2. The $\mathrm{T}_{1}$ excited-state spectrum extracted according the Equation (1),

$\chi_{T_{1}}(k)=\frac{\chi(k)_{\text {laser-on }}-\chi(k)_{\text {laser-off }} \cdot 50 \%}{50 \%}$

where $k$ is the photon electron wave vector, $\chi(k)_{\text {laser-off }}$ and $\chi(k)_{\text {laser-on }}$ are from the EXAFS spectra in Figure 3 of the main text, and $\chi(k)_{T 1}$ is the resulting signal for the $T_{1}$ excited state. $\chi(k)_{\text {laser-off }}$ and $\chi(k)_{T 1}$ are used for the ground-state and the $\mathrm{T}_{1}$ excited-state spectra, respectively. The Fourier transformed EXAFS spectra with $k^{2}$ weighting are respectively used for the fitting shown in Figure 2. The FEFF parameters were calculated from the crystal structure. $^{2}$ The goodness of the agreement between the experimental spectrum and the calculated spectrum with the fitting parameters are also shown in Figure 2. The same $k$ ranges (1.8-8.5) were used in fitting both spectra. Only the nearest two neighboring shells of the Ni were fit because the signal to noise ratio of the data for the excited state was not considered sufficient to extract structural parameters for more distant neighbors. The fitting results are shown in Figure 2. The precision for the distances $R$ is around $0.02 \AA$. The coordination numbers $\mathrm{N}$ were fixed during the fitting.
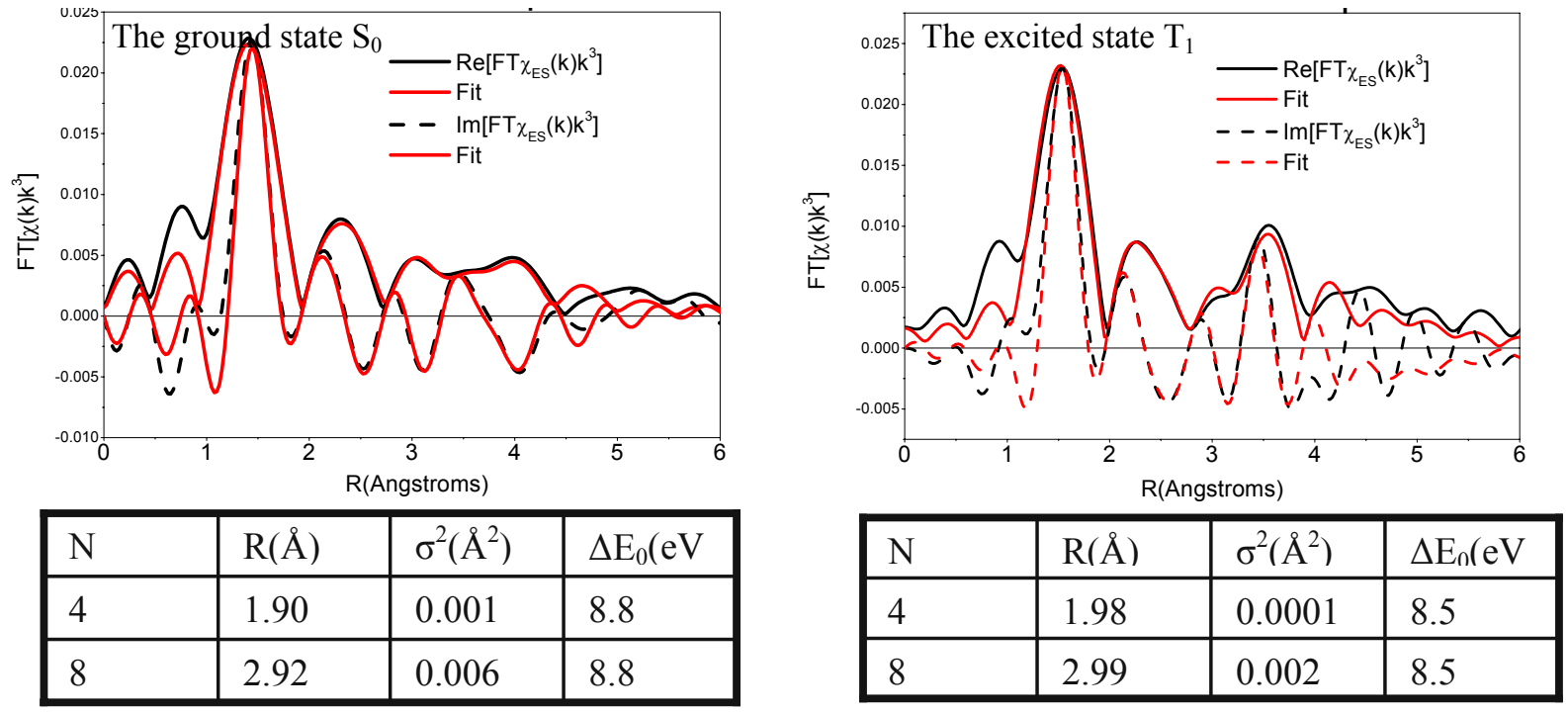

Figure 2. The fitting curves for the ground state $\mathrm{S}_{0}$ (left) and the excited state $T_{1}$ (right) with the corresponding structural parameters shown in the tables under the figures. 


\section{References}

(1) Jennings, G., Jaeger, W. J. H., Chen, L. X., Rev. Sci. Instr. 2002, 72, 364-368.

(2) McLean, A. L.; Foran, G. J.; Kennedy, B. J.; Turner, P.; Hambley, T. W., Aust. J. Chem. 1996, 49, 1273-1278. 